\title{
O impacto da neurociência na identidade profissional do professor que ensina
} matemática

\author{
Marcos Guilherme Moura-silva*
}

\section{Resumo}

O advento da Neurociência e sua evolução ao longo das últimas décadas ampliaram as compreensões acerca do funcionamento cerebral, trazendo implicações importantes para o campo de inquérito da educação matemática e formação de professores. Por outro lado, muitos dos conhecimentos neurocientíficos sobre como o cérebro aprende matemática não se refletem na prática dos professores e consequentemente em suas identidades profissionais. Este ensaio tensiona discutir o impacto que a neurociência cognitiva pode trazer para a identidade do professor que ensina matemática e como isso pode estabelecer mudanças paradigmáticas em suas práticas pedagógicas. Para tanto, conceituo "identidade docente" destacando o papel do discurso, do contexto e da comunidade de prática integradas por professores e neurocientistas como eixos de impacto na identidade profissional. Em seguida, discuto como resultados neurocientíficos podem influenciar a prática profissional docente a partir das três classificações propostas por Smedt e Grabner (2015), a saber: conhecimento neurológico, neuropredição e neurointervenção. Concluo advogando a implementação de uma perspectiva investigativa para a área de formação de professores que integre aspectos comportamentais, cognitivos e fisiológicos em relação à aprendizagem matemática, indicando a importância de uma alfabetização neurocientífica para o desenvolvimento profissional docente.

Palavras-chave: Identidade Docente; Neurociência Cognitiva; Formação de Professores.

* Doutor em Educação em Ciências e Matemática. Docente da Universidade Federal do Pará-UFPA, Brasil. Instituto de Educação Matemática e Científica-IEMCI. Brasil. E-mail: marcosgmouras@yahoo.com.br 


\section{Introdução e propósito}

O advento da Neurociência e sua evolução ao longo das últimas décadas ampliaram as compreensões acerca do funcionamento cerebral, trazendo implicações importantes para o campo educacional (KATZIR E BLAGOEV, 2006; GOSWAMI, 2006; ANSARI, COCH e SMEDT, 2011). O uso de tecnologias de neuroimagem (fMRI) e métodos como Eletroencefalograma (EEG), Tomografia por emissão de Pósitron (TEP), Medidas Psicofisiológicas- relativas a mudanças fisiológicas ocasionadas por processos emocionais, possibilitaram desvendar os distintos processos cognitivos, em especial a aprendizagem humana (ANSARI, 2008; ver Dick et al., 2014 para introdução sobre métodos neurocientíficos para pesquisa educacional)

No que concerne ao campo de inquérito da Educação Matemática, diversas frentes de investigação foram desbravadas, trazendo entendimentos acerca da cognição matemática. Por exemplo, estudos de neuroimagem tem sido focados no desenvolvimento de habilidades aritméticas em crianças e adultos (ZAMARIAN et al., 2009), enquanto outros vem situar as regiões cerebrais responsáveis pelo cálculo mental (ver DE SMEDT et al., 2010; GRABNER et al., 2013). Baseados em estudos de imagens cerebrais, algumas investigações vêm propondo modelos voltados à proficiência Matemática em problemas algébricos, a partir de ações coordenadas de várias regiões cerebrais (ANDERSON et. al., 2008), e outros vem destacando o rastreamento ocular como método promissor para medir estratégias de resolução de problemas (OBERSTEINER e TUMPEK, 2016)

Revisão Sistemática realizada por Yen Looi e colaboradores (2016), procurou fornecer uma visão geral do progresso no campo da neurociência cognitiva acerca da compreensão da aprendizagem matemática, destacando linhas temáticas de investigação, como o desenvolvimento da cognição numérica em humanos (IZARD et al., 2009; GEARY et al., 2000; CAMPBELL e ALBERTS, 2009), estudos situados na aprendizagem aritmética (DEHAENE, 2009; DELAZER et al., 2003; GRABNER et al., 2009; ISCHEBECK et al., 2009;), investigações focadas na ansiedade matemática (DOWKER et al., 2012; MALONEY e BEILOCK, 2012; MOURA-SILVA et al., 2019) além de estudos feitos em populações especiais, como aquelas que apresentam acalculia ( HUMPHREYS et al., 2012; DELAZER et al., 2004) e Discalculia Desenvolvimental (LANDERL et al., 2004; KUCIAN et al., 2013; RYKHLEVSKAIA et al., 2009). Outras investigações revelam que os distintos contextos emocionais ativam diferentes 
aspectos da memória (BUCHANAN e TRANEL, 2008; KENSINGER e SCHACTER, 2008), possibilitando, desse modo, aprofundar questões envolvendo emoção e cognição.

Em contraponto à essa evolução, percebe-se que muitos dos conhecimentos neurocientíficos sobre como o cérebro aprende matemática não se refletem nas práticas dos professores, e, portanto, não fazem parte de suas identidades e desenvolvimento profissionais. Estudo conduzido por Grossi, et al., (2019), constatou que no Brasil, ainda é pequena a influência da neurociência na sala de aula e nos cursos de formação de professores e que nada mudou ao longo da última década. Frente a isso, pergunta-se: não é curioso e ao mesmo tempo alarmante que o cérebro, enquanto órgão privilegiado por onde ocorre a aprendizagem, seja o principal instrumento de trabalho do professor e dele pouco se conheça? Quais poderiam ser os impactos dos conhecimentos neurocientíficos na identidade profissional do professor de matemática? Neste ensaio, trago reflexões acerca disso e indico possíveis caminhos para implementação de uma perspectiva investigativa para a área de formação de professores que integre aspectos comportamentais, cognitivos e fisiológicos em relação à aprendizagem matemática.

Para o escopo deste artigo, discuto a seguir alguns dos fatores que impactam a identidade do professor, segundo a literatura da área, destacando o discurso, o contexto e a prática. Na sequência, incluo a neurociência cognitiva como uma ferramenta capaz de influenciar esses fatores, impactando consequentemente a identidade docente. Discuto três caminhos possíveis por onde os conhecimentos neurocientíficos podem infundir o pensamento do professor e concluo defendendo a importância de uma perspectiva sistêmica de investigação que inclua o fator biológico da aprendizagem como eixo de desenvolvimento profissional do professor que ensina matemática.

\section{Identidade docente e fatores de impacto}

As últimas duas décadas foram marcadas pelo expressivo aumento das investigações sobre identidade do professor e diferentes lentes de abordagens foram mostradas na literatura como fatores que produzem impactos nessa identidade, a saber: o fator das narrativas e do discurso, o fator dos fatores contextuais e o fator das comunidades de prática (BEUCHAMP e THOMAS, 2009). Discuto brevemente sobre cada um deles, a seguir. 
O primeiro fator, encontra nas "narrativas e no discurso", uma via de acesso por onde o professor constitui uma identidade profissional. "Evidentemente, as narrativas dos professores sobre si mesmos e suas práticas, bem como os discursos que os envolvem, oferecem oportunidades para explorar e revelar aspectos de sua identidade" (BEUCHAMP e THOMAS, 2009, p. 181, tradução própria). Assim, parafraseando Sfard e Prusak, (2005), “identidades" são coleções de histórias pessoais que reifica, endossa e traz significado, sendo, portanto, uma via de constituição identitária (ver também OLIVEIRA, 2012; WATSON, 2006). Conectada à noção de narrativa, está o do discurso, pelo qual o indivíduo revela sua identidade e a negocia em diferentes contextos (BEYNON, 1997). Os discursos pelos quais os professores se envolvem, contribuem para a formação de suas identidades e podem exercer poder transformador em suas ações. Nesse propósito, Alsup (2006) mostrou que através de um engajamento discursivo, professores em formação expandiram suas identidades profissionais, provocando transformações em seus pensamentos. Esses discursos, segunda a autora, estão relacionados a linguagens, ações, emoções ou ideias nos quais os professores estão envolvidos. Desse modo, o discurso, seja interno ou externo, tem profundo impacto na formação da identidade docente.

Um segundo fator analítico pelo qual a identidade do professor pode sofrer influência, é o fator do contexto. Vários estudos vêm mostrando que os fatores contextuais (incluindo os objetos de saber ou disciplina de ensino) influenciam a construção da identidade docente e seu desenvolvimento profissional (FREITAS, 2008; DRAKE et al., 2001; GOOS e BENNISON, 2008; HOBBS, 2012; LUTOVAC e KAASILA, 2014; OWENS, 2014). O ambiente escolar, o impacto com outros profissionais e os próprios programas de formação de professores exercem impactos nessa constituição identitária, com influências diretas nas experiências professorais.

Um terceiro fator analítico tem a ver com comunidades de prática. Na perspectiva sociocultural, como a informada nos estudos sobre trabalho e aprendizagem situada em comunidades de prática - amplamente discutida em Wenger (1998)- encontramos um vínculo da identidade docente à sua prática. Para Wenger (1998 p. 149), "a identidade é a experiência negociada de si mesmo, envolve a participação em comunidade, tem uma trajetória de aprendizagem, combina diferentes formas de associação dentro de uma identidade e presume envolvimento em contextos locais e globais". Participando de uma comunidade de profissionais, o professor sujeita-se as influências desta comunidade em sua constituição identitária. 
Frente a isso e baseado em recente levantamento (GROSSI et al., 2019), percebemos que em nenhum desses três fatores de impacto identitário: discurso, contexto e prática, o saber neurocientífico está sendo envolvido efetivamente na formação do professor. Contudo, não é raro encontrarmos a difusão de neuromitos- concepções popularmente sustentadas, mas confusas, da função cerebral (VAN DER MEULEN et al., 2015 )- nas práticas docentes (DEKKER et al., 2012). Um exemplo de neuromito é que a aprendizagem poderia ser melhorada se crianças fossem classificadas e ensinadas conforme seu estilo de aprendizagem preferido. Embora resultados empíricos validem esse tipo de afirmação: a de que informações visuais, auditivas e cinestésicas podem ser processadas em diferentes partes do cérebro, sabe-se que essas estruturas cerebrais estão altamente interconectadas (GILMORE et al., 2007), não havendo melhora significativa de aprendizagem quando crianças são educadas conforme seu estilo de aprendizagem (COFFIELD et al., 2004).

Assim, torna-se imperativo a inserção correta de evidências neurocientíficas, via discurso teórico e prático no desenvolvimento profissional do professor; a partir da reformulação de contextos formativos iniciais ou continuados, que abranjam a área da neurociência cognitiva; e através da desejável colaboração de professores e neurocientistas em comunidades de prática, visando ampliar as competências profissionais e coordenar ações do professor em sala de aula. Algumas experiências já foram relatadas nesse sentido (por exemplo, TAN e AMIEL, 2019), sugerindo que a neurociência moldou as práticas de ensino de maneira profunda, contribuindo para uma tomada de decisão pedagógica pautadas em entendimentos claros do funcionamento cerebral. Baseado em Smedt e Grabner (2015), apresento três possibilidades pelas quais os resultados neurocientíficos podem infundir e impactar a prática do professor de matemática em sala de aula e como isso pode influenciar sua identidade profissional.

Neurociência e a prática do professor de matemática

A última década testemunhou um avanço exponencial de pesquisas do cérebro relacionadas à cenários educacionais. Surgia assim, um campo de pesquisa interdisciplinar e translacional integrando mente, cérebro e educação (ver FISHER, 2009), também denominado de neuroeducação ou neurociência educacional, com objetivo central de encontrar assinaturas neurais para a aprendizagem (por exemplo, HOWARD-JONES, 2010, GOSWAMI e SZÜCS, 2011). Contudo, por falta de um paradigma de pesquisa claro ou fundamentos filosóficos sólidos, essa iteração não 
obteve pleno êxito em seu início de desenvolvimento, ao ponto de ser considerada uma "ponte longe demais" (BRUER, 1997) de ser construída.

Apesar disso, a neurociência educacional avançou e constitui-se atualmente uma forte ferramenta para aumentar nossas capacidades explicativas e preditivas para as teorias da educação matemática (LEIKIN, 2018), fato pelo qual se hipotetiza que também pode haver impactos na identidade docente. No presente texto, ressalto três possibilidades pelas quais a neurociência pode ser infundida na prática do professor que ensina matemática, baseadas nas classificações feitas por Smedt e Grabner (2015), sendo elas: a compreensão neurológica, a neuropredição e a neurointervenção. A compreensão neurológica, refere-se à capacidade da pesquisa neurocientífica aprofundar o entendimento do processamento matemático em um nível biológico, informando o professor sobre o desenvolvimento típico e atípico de competências matemáticas ensinadas na escola. A neuropredição ou neuroprognose, possibilita a oportunidade de refletir sobre os resultados neurocientíficos numa perspectiva de prever trajetórias de aprendizagem ou respostas a intervenções educacionais. A neurointervenção, refere-se ao uso reflexivo dos resultados neurocientíficos para fundamentar intervenções educacionais ou mensurar seus efeitos no desempenho e na aprendizagem matemática. Visando exemplificar cada uma dessas classificações, apresento análises de evidências cerebrais para cada uma delas.

\section{Compreensão neurológica}

As evidências neurocientíficas podem fornecer ao professor complementações sobre as aquisições de habilidades matemáticas, estendendo conhecimentos advindos da psicologia e de teorias educacionais. Um exemplo, nessa perspectiva, tem a ver com as redes neurais de processamento aritmético e como o déficit em alguns dos componentes dessa rede, pode acarretar deficiências severas no aprendizado aritmético, como a discalculia, por exemplo.

Sabe-se que a organização neural da aritmética é dinâmica e que sub-redes se alternam conforme o processo de aprendizagem (BUTTERWORTH, 2011, PETERS e DE SMEDT, 2018). Por exemplo, a apreensão de fatos novos de aritmética envolve regiões como os lobos frontais e os sulcos intraparietais, enquanto o uso desses fatos em outros contextos, envolve o giro angular esquerdo, que está implicado na recuperação de fatos da memória (ISCHEBECK et al., 2009). Uma 
vez que as evidências demonstram que os sulcos intraparietais estão envolvidos em manipulação ou representação de números, o desenvolvimento atípico dessa região acarretará prejuízos nessas funções cognitivas e consequentemente afetará habilidades numéricas simples e complexas (figura 1). É exatamente o que vem sendo sugerido para indivíduos com discalculia, onde foi observado atividade neural reduzida justamente na região dos sulcos intraparietais (ROTZER et al., 2008; RYKHLEVSKAIA et al., 2009). Como consequência comportamental, os números não parecem ser significativos para indivíduos com esse tipo de desordem cognitiva, de modo que eles não entendem de modo intuitivo a magnitude de um número e não conseguem estabelecer relações com outros. Mais recentemente, Peters e De smedt (2018), revisaram essa rede aritmética, incluindo áreas pré-frontal, parietal posterior, ocipito-temporal e hipocampal, indicando que essa rede passa por mudanças de desenvolvimento em relação a sua função, conectividade e estrutura, que ainda precisam ser melhor exploradas.

Figura 1 - À esquerda, um modelo causal proposto por Butterworth (2011) estabelecendo uma relação entre aspectos biológicos, cognitivos e comportamentais em relação ao aprendizado aritmético. À direita, ilustração discutida em peters e de smedt (2018), ampliando as áreas implicadas na aritmética de maneira mais relevante, incluindo DLPFC = córtex pré-frontal dorsolateral, $\mathrm{VLPFC}=$ cortes pré-frontais ventrolaterais, $\mathrm{HC}=$ hipocampo, $\mathrm{PSPL}=$ lobo parietal superior posterior,$I P S=$ sulco intraparietal,$S M G=$ giro supramarginal,$A G$ = giro angular e FG = giro fusiforme. Imagens extraídas de BUTTERWORTH, 2011, p.X e de PETERS e DE SMEDT , 2018, p.
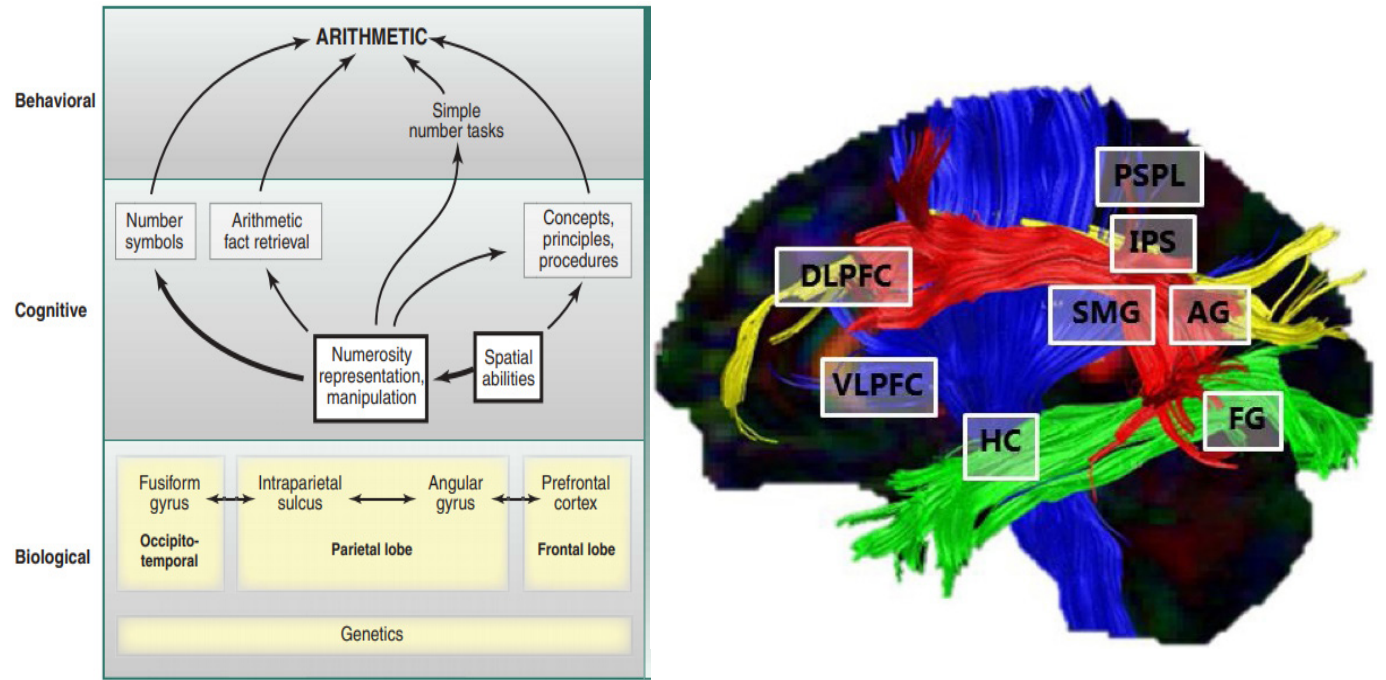
Tais resultados, podem possibilitar ao professor um aprofundamento biológico da aprendizagem matemática, que poderá propiciar impactos em suas abordagens pedagógicas. Contudo, a natureza desse impacto (se positivo ou negativo), dependerá da forma como o conhecimento neurocientífico é percebido e conduzido junto ao professor. Nesse ponto, é importante destacar que não vislumbro a neurociência como a panaceia que resolverá todos os problemas relativos à aprendizagem matemática e tampouco terá efeito prático sem uma reflexão advinda da experiência profissional docente. A compreensão neurológica só fará sentido ao professor ao ponto de gerar impactos em sua identidade profissional se ela for comunicada e baseada em cenários educacionais e exercida numa perspectiva interdisciplinar, em mão dupla.

\section{Neuropredição ou neuroprognose}

Neuropredição ou neuroprognose estabelece os resultados neurocientíficos como ferramenta que pode ser usada para prever resultados comportamentais e educacionais. Especificamente em relação ao campo da aprendizagem matemática, Supekar et al. (2013) investigou quais preditores comportamentais e neurais poderiam levar crianças de 8 e 9 anos a ter maiores habilidades aritméticas que outras, considerando uma tutoria cognitiva de 8 semanas. Variáveis comportamentais (como habilidades aritméticas, QI, leitura, memória de trabalho e habilidades matemáticas), além de dados de imagem cerebral (fMRI) foram desenvolvidas antes da intervenção de tutoria. Após isso, supekar e colaboradores (2013), analisaram quais de suas variáveis (se as comportamentais ou imagens cerebrais) eram capazes de prever o desempenho aritmético. Seus dados revelaram que somente as medidas de fMRI foram capazes de prever as habilidades aritméticas sob estudo, sugerindo que dados de neuroimagem podem ser biomarcadores confiáveis para identificar crianças com risco de baixo desempenho em estágio inicial. Educacionalmente, a descoberta de tais marcadores neurais possibilitaria ao professor compreender como está o desenvolvimento matemático do aluno antes de uma instrução formal ou como ocorrem suas diferentes trajetórias de desenvolvimento.

Mais recentemente, as descobertas de Moura-Silva et al., (2019), também possibilitou encontrar marcadores biológicos para ansiedade matemática- um transtorno emocional de aprendizagem, na variabilidade da frequência cardíaca (VFC) em crianças escolares. Os autores descobriram que em condições de repouso, 
a complexidade da VFC é aumentada em crianças com alta ansiedade matemática e que esta complexidade diminui quando este mesmo grupo está sob condição de estresse, como um teste de aritmética mental, por exemplo. Tais resultados, podem orientar o trabalho do professor no sentido de avaliar e gerenciar um transtorno de aprendizagem matemático específico, como a ansiedade matemática, em contextos escolares, a partir de um sinal biológico mais facilmente manipulável que aqueles relacionados à imagem cerebral.

\section{Neurointervenção}

A neurointervenção refere-se ao uso reflexivo dos resultados neurocientíficos para informar intervenções educacionais ou mensurar seus efeitos no desempenho e na aprendizagem matemática. O cuidado com o uso de tais conhecimentos neurocientíficos, entretanto, deve ser observado, no sentido de que não seja prescritivo, mas sugestivo ao ponto de ser combinado com princípios pedagógicos. Há vários relatos de neurointervenção aplicados à desenvolvimentos matemáticos atípicos, como a discalculia, dos quais fizeram uso de softwares capazes de gerar significado numérico ou treinar magnitudes numéricas que são alterados nessa população, conforme discutido anteriormente.

Outras abordagens de neurointervenção, são baseadas em método de estimulação elétrica trasncraniana (EET). No EET, uma pequena corrente elétrica (normalmente cerca de 1-2 mA) não invasiva é aplicado no cérebro através de eletrodos fixados no couro cabeludo. Tal corrente tem a função de alterar níveis de ativação de regiões corticais. Cohen Kadosh et al. (2010) examinaram em um grupo de universitários se a EET aplicada na região parietal poderia influenciar de maneira benéfica a aquisição de símbolos numéricos fictícios. Descobriu-se que a estimulação de 20 minutos diárias antes de sessões de aprendizagens resultou em maior automação procedimental, gerando efeitos de aprendizagem de longo prazo nos estudantes que receberam estimulação Transcraniana. Ressalta-se, entretanto, que nesse caso, o uso desses resultados na sala de aula se torna mais limitados.

Em contrapartida ao estudo anterior, os relatórios de Lee et al. (2007, 2010), apresentaram resultados mais ecológicos para sala de aula. Em tais estudos, investigou-se se os processos cognitivos implicados na solução de um problema algébrico são os mesmos quando se usa expressões algébricas ou representação pictórica 
(modelos esquemáticos). Dezoito participantes, proficientes em ambas as estratégias de resolução, solucionaram o problema via equação e via modelo esquemático, constatando-se a ativação de áreas vinculadas a memória de trabalho e procedimento quantitativo. Os autores concluíram que ambas as estratégias usam processos cognitivos semelhantes, mas o método de equação impôs maior demanda de atenção ao cérebro quando comparada ao método do modelo esquemático. Tal evidência, pode ser considerada útil ao professor que ensina matemática sobre quando e como tais métodos podem ser introduzidos em sala de aula.

Outro resultado interessante, foi desenvolvido por Babai et al., (2016), que investigaram os efeitos de uma intervenção pedagógica para o ensino de perímetro de figuras planas, baseando-se na evidência da variável relevante. Anteriormente, um estudo de imagem cerebral relacionado à tarefa de comparação de perímetros sugeriu que aumentar o nível de saliência da variável relevante, perímetro, aumentaria o desempenho dos participantes (Stavy, 2006). Pautados em tais evidências, autores aumentaram o nível de saliência da variável relevante (perímetro), cobrindo as figuras com várias unidades de palitos de fósforo, chamadas pelos autores de modo de apresentação discreta, em contraste com o modo de apresentação contínua, que consistia em desenhá-los continuamente no papel (figura 2 ).

Figura 2 - Intervenção Didática baseada em resultados neurocientíficos- o uso de apresentações discretas e contínuas em relação ao ensino de perímetro de figuras planas
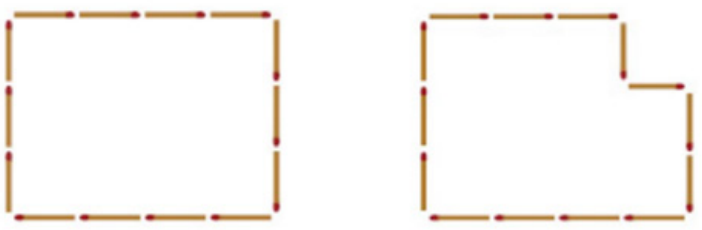

A) Modo de apresentação discreto
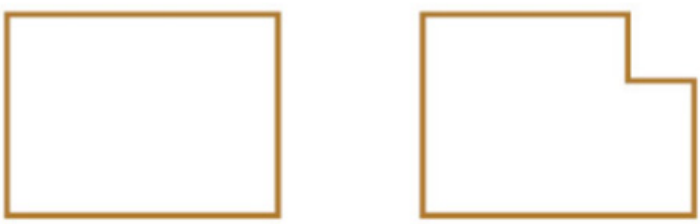

B) Modo de apresentação contínuo 
O aumento de saliência, segundo os autores, atraiu a atenção dos participantes e oportunizou o uso de estratégias comumente utilizadas na resolução de tarefas dessa natureza, como mover/contar segmentos ou aplicar conhecimentos geométricos. Os resultados indicaram aumento de desempenho quando os alunos foram expostos ao modo de apresentação discreto, e sucesso no modo contínuo após terem tido contato com a primeira intervenção (ou seja, após realizada as tarefas de modo discreto). Resultados dessa natureza, evidenciam uma conexão producente entre educação matemática e neurociência, informando qual o melhor modo ou ordem de apresentação de problemas para os alunos, impactando sobremaneira o modo como o professor conduz ou planeja suas aulas.

\section{Conclusão}

Como principal instrumento de trabalho do professor, é surpreendente que o conhecimento sobre como o cérebro aprende matemática não esteja efetivamente presente na identidade profissional, revelada pelo discurso, pelos contextos formativos ou pelas práticas de ensino. Como discutido, os resultados neurocientíficos, combinados com resultados comportamentais e educacionais podem possibilitar práticas mais amplas e informadas, encontrando respaldos científicos para além dos saberes experienciais. A aprendizagem matemática, como um processo mental e emocional, não pode ser considerada isoladamente de nossa fisiologia, mas, pelo contrário, deve ser entendida como parte integrante das diversas funções interativas e complexas que compõe o organismo do aprendente. Como já informado em Dubinsky, Roehrig e Varma ( 2013 ), uma mudança ontológica na forma como professores concebem a aprendizagem humana tem a capacidade de mudar fundamentalmente a forma como os educadores abordam a preparação de aulas e o projeto curricular. Torna-se necessário uma alfabetização neurocientífica, para além das formações psicológicas e educacionais, de modo a averiguarmos os impactos reais que a neurociência poderá trazer para o professor e para seu desenvolvimento profissional. 


\section{The impact of neuroscience on the professional identity of the teacher who teaches mathematics}

\section{Abstract}

The advent of Neuroscience and its evolution over the last decades have broadened the understanding of brain functioning, bringing important implications for the field of inquiry in mathematics education and teacher training. On the other hand, much of the neuroscientific knowledge about how the brain learns mathematics is not reflected in the practice of teachers and consequently in their professional identities. This essay tends to discuss the impact that cognitive neuroscience can bring to the identity of the teacher who teaches mathematics and how it can establish paradigmatic changes in their pedagogical practices. For that, I conceptualize "teaching identity" highlighting the role of discourse, context and the community of practice integrated by teachers and neuroscientists as axes of impact on professional identity. Then, I discuss how neuroscientific results can influence professional teaching practice based on the three classifications proposed by Smedt and Grabner (2015), namely: neurological knowledge, neuroprediction and neurointervention. I conclude by advocating the implementation of an investigative perspective for the area of teacher education that integrates behavioral, cognitive and physiological aspects in relation to mathematical learning, indicating the importance of neuroscientific literacy for the professional development of teachers.

Keywords: Teaching identity; Cognitive Neuroscience; Teacher training.

ALSUP, Janet. Teacher identity discourses: Negotiating personal and professional spaces. Teacher identity Discourses, 1- 234, outubro, 2006. doi: 10.4324 / 9781410617286

ANSARI, Daniel. Effects of development and enculturation on number representation in the brain. Nature Reviews Neuroscience, 9(4):278-291, abril, 2008. doi:10.1038/nrn2334.

ANSARI, Daniel; COCH, Donna e DE SMEDT, Bert. Connecting Education and Cognitive Neuroscience: Where will the Journey Take us? Educational Philosophy and Theory. 43 (1): 36-41, fevereiro, 2011. doi: 10.1002/9781444345827.ch6.

BABAI, Reuven., NATTIV, Laura., e STAVY, Ruth. Comparison of perimeters: improving students' performance by increasing the salience of the relevant variable. ZDM: the international journal on mathematics education.48 (3): 379-383, Junho, 2016. doi:10.1007/ s11858-016-0766-z.

BEAUCHAMP, Catherine.; THOMAS, Lynn. Understanding teacher identity: an overview of issues in the literature and implications for teacher education. Cambridge Journal of Education, Abingdon, v. 39, n. 2, p. 175-189, Maio, 2009.

BEYNON, Carol. Crossing over from student to teacher: Negotiating an identity. Canadá. 1997. Tese de Doutorado. Department of Education. University of Toronto, 1997.

BRUER, John. T. Education and the brain: a bridge too far. Educational Researcher, 26(8), 4-16, setembro, 1997. 
BUCHANAN Tonny W, TRANEL Daniel. Stress and emotional memory retrieval: effects of sex and cortisol response. Neurobiol Learn Mem. 89(2):134-141, fevereiro, 2008. doi: 10.1016/j. nlm.2007.07.003

BUTTERWORTH, Brian., VARMA, Sachank.; LAURILLARD, Diana. Dyscalculia: from brain to education. Science, 332 (6033), 1049-1053. Maio, 2011. doi: 10.1126 / science.1201536

CAMPBELL, Jamie ID; ALBERTS, Nicole M. Operation-specific effects of numerical surface form on arithmetic strategy. Journal of Experimental Psychology: Learning, Memory, and Cognition, 35(4): 999-1011, Agosto, 2009. doi: 10.1037 / a0015829

COFFIELD, Frank; MOSELEY, David; HALL, Elaine; ECCLESTONE, Kathryn. Learning Styles and Pedagogy in Post-16 Learning. A Systematic and Critical Review. London: Learning and Skills Research Centre. Disponível em: http://evidence.thinkportal.org/handle/123456789/62, acesso em: 20/09/2020., 2004.

DE SMEDT, Bert., HOLLOWAY, Ian. D.; ANSARI, Daniel. Effects of problem size and arithmetic operation on brain activation during calculation in children with varying levels of arithmetical fluency. Neuroimage 57(3): 771-781, Dezembro, 2010.

DE SMEDT, Bert; GRABNER, Roland H. Applications of Neuroscience to Mathematics Education. The Oxford Handbook of Numerical Cognition, p.1-17, novembro, 2014. doi: 10.1093 / oxfordhb / 9780199642342.013 .48

DEHAENE, Stanislas. Origins of mathematical intuitions: the case of arithmetic. Annals of the New York Academy of Sciences, Vol. 1156 (1): 232-259, Março, 2009. doi: 10.1111 / j. 1749-6632.2009.04469.x.

DEKKER Sanne, LEE Nikki C; HOWARD-JONES Paul; JOLLES Jelle. Neuromyths in education: Prevalence and predictors of misconceptions among teachers. Front. Psychology 3:429. agosto, 2012. doi: 10.3389/fpsyg.2012.00429

DELAZER, M; DOMAHS, F; BARTHA, L; BRENNEIS, C; LOCHY, A; TRIEB, T; BENKE, T. Learning complex arithmetic - an fMRI study, Cognitive Brain Research, 18(1): 76-88, Dezembro, 2003. doi: https://doi.org/10.1016/j.cogbrainres.2003.09.005

DELAZER, Margarete, DOMAHS , Frank; LOCHY , Aliette ; KARNER , Elfriede; BENKE , Thomas; POEWE, Werner. Number processing and basal ganglia dysfunction: A single case study, Neuropsychologia, Vol. 42(8): 1050-1062, 2004. doi: 10.1016/j.neuropsychologia.2003.12.009

DICK, F., LLOYD-Fox, S., BLASI, A., ELWELL, C., MILLS, D. Neuroimaging methods. In D. Mareschal, B. Butterworth, \& A. doctoral dissertation, OISE/UofT, 2014.

DOWKER, Ann.; BENNETT, Karina; SMITH Louise. Attitudes to Mathematics in Primary School Children, Child Development Research, 8(2012), novembro, 2012. doi: 10.1155 / 2012/124939

DRAKE, Corey.; SPILLANE, James. P.; HUFFERD-ACKLES, Kimberly. Storied identities: teacher learning and subject-matter context. Journal of Curriculum Studies, Abingdon, 33(1): 1-23, 2001. DOI: https://doi.org/10.1080/00220270119765.

DUBINSKY, Janet, M; ROEHRIG, Gillian e VARMA, Sashank . Infusing Neuroscience Into Teacher Professional Development. Educational Researcher 42(6):317-329, Agosto, 2013. doi: 10.3102/0013189X13499403.

FISCHER, Kurt. W. Mind, brain, and education: building a scientific groundwork for teaching and learning. Mind, Brain and Education, 3(1): 3-16, março, 2009. 
FREITAS, Elisabeth. Troubling teacher identity: preparing mathematics teachers to teach for diversity. Teaching Education, Abingdon. 19 (1): 43-55, março, 2008. doi: 10.1080 / 10476210701860024

GEARY, David. C. "From infancy to adulthood: The development of numerical abilities", European child and adolescent psychiatry, Vol. 9(2): S11-S16, junho, 2000. disponível em: https://web. missouri.edu/ gearyd/ECAPsychiatry.pdf, acesso em 20/09/2020.

GILMORE, Camila. K.; MCCARTHY, Shannon. E.; SPELKE, Elizabeth. S. Symbolic arithmetic knowledge without instruction. Nature 447, 589-592, maio, 2007.

GOOS, Merrilyn. E.; BENNISON, Anne. Developing a communal identity as beginning teachers of mathematics: emergence of an online community of practice. Journal of Mathematics Teacher Education, Dordrech, 11(1):41-60, Dezembro, 2008.

GOSWAMI, Usha; SZÜCS, Dénes. Educational neuroscience: developmental mechanisms: towards a conceptual framework. Neuroimage, 57(3): 651-658, agosto, 2011. Doi:10.1016/j. neuroimage.2010.08.072

GOSWAMI, Usha. Neuroscience and education: from research to practice? Nature Reviews of Neuroscience. 7(5): 406-413, abril, 2006.

GRABNER, Roland. H.; ANSARI, Daniel.; KOSCHUTNIG, Karl., REISHOFER, Gernot.; EBNER, Franz. The function of the left angular gyrus in mental arithmetic: evidence from the associative confusion effect. Hum. Brain. Mapp. 34 (5): 1013-1024, Maio 2013. doi: 10.1002/hbm.21489. Epub

GRABNER, Roland.H; ANSARI, Daniel; KOSCHUTNIG, Karl REISHOFER, Gernot EBNER, Franz; NEUPER, Christa . To retrieve or to calculate? Left angular gyrus mediates the retrieval of arithmetic facts during problem solving, Neuropsychologia, Vol. 47(2): 604-608, janeiro, 2009. doi: http://dx.doi.org/10.1016/j.neuropsychologia.2008.10.013.

HOBBS, Linda. Teaching 'out-of-field' as a boundary-crossing event: factors shaping teacher identity. International Journal of Science and Mathematics Education, Dordrecht, v. 11, n. 2, p. 271-297, Agosto, 2012.

HOWARD-JONES, Paul. Introducing Neuroeducational Research. Neuroscience, Education, and the Brain from Contexts to Practice. London: Routledge, 2010.

HUMPHREYS, Glyn. W. et al. BCoS: Brain behaviour analysis, Psychology Press, London. 2012.

ISCHEBECK Anja; ZAMARIAN Laura; SCHOCKE Michael; DELAZER Margarete. Flexible transfer of knowledge in mental arithmetic--an fMRI study. Neuroimage, 44(3),1103-1112, fevereiro 2009. doi:10.1016/j.neuroimage.2008.10.025.

ISCHEBECK, Anja; SCHOCKE, Michael; DELAZER, Margarete. The processing and representation of fractions within the brain: An fMRI investigation, NeuroImage, 47(1), 403-413, abril 2009. doi: 10.1016 / j.neuroimage.2009.03.041.

IZARD Véronique; SANN, Coralie; SPELKE Elizabeth; STRERI Arlette. Newborn infants perceive abstract numbers, Proceeding of the National Academy of Science USA, 106(25):1038210385, junho 2009. doi: 10.1073/pnas.0812142106

KATZIR, Tami; PARÉ-BLAGOEV, Juliana. Applying cognitive neuroscience research to education: The case of literacy. Educational Psychologist, 41(1), 53-74, Março 2006. doi.org/10.1207/ s15326985ep4101_6 
KENSINGER EA, SCHACTER DL. LEWIS M, HAVILAND-JONES JM, BARRETT LF. Memory and emotion. APA PsycNet, Handbook of Emotions, Guilford Press 601-617, 2008

KUCIAN, Karin; ASHKENAZI, Simone Schwizer; HÄNGGI Jürgen; ROTZER Stephanie; JÄNCKE Lutz; MARTIN Ernst; ASTER, von Aster. Developmental dyscalculia: A dysconnection syndrome?. Brain Structure and Function, 219 (5), 1721-33, Setembro 2014. doi.org/10.1007/ s00429-013-0597-4.

LANDERL, Karin; BEVAN, Anna; BUTTERWORTH, Brian. Developmental dyscalculia and basic numerical capacities: A study of 8-9-year-old students". Cognition, 93(2),99-125, outubro 2004. doi: 10.1016/j.cognition.2003.11.004.

LEE, Kerry; LIM, Zee Ying; YEONG, Stephanie.H.M., Ng, Swee Fong; VENKATRAMAN, Vinod; CHEE, Michael WL. Strategic differences in algebraic problem solving: neuroanatomical correlates. Brain Research, 1155,163-171, junho, 2007.doi.org/10.1016/j.brainres.2007.04.040.

LEE, Kerry; YEONG, Stephanie.H.M., Ng, Swee Fong; VENKATRAMAN, Vinod. Computing solutions to algebraic problems using a symbolic versus a schematic strategy. ZDM: The International Journal on Mathematics Education, 42(6), 591-605, Outubro de 2010. doi: 10.1007/ s11858-010-0265-6.

LEIKIN R. How Can Cognitive Neuroscience Contribute to Mathematics Education? Bridging the Two Research Areas. n book: Invited Lectures from the 13th International Congress on Mathematical Education (pp.363-383) Project: Implementing Neuroscience tools to Mathematics Education Research. Janeiro, 2018. DOI: 10.1007/978-3-319-72170-5_21.

LOOI, Yen hung; THOMPSON, Jacqueline; KRAUSE, Beatrix; KADOSH, Roi Cohen. The Neuroscience of Mathematical Cognition and Learning. OECD Education Working Papers 136, OECD Publishing, 2016. doi: 10.1787 / 5jlwmn3ntbr7-en.

LUTOVAC, Sonja; KAASILA, Raimo. Pre-service teachers' future-oriented mathematical identity work. Educational Studies in Mathematics, 85: 129 - 142, julho 2014.

MALONEY, Erin A; BEILOCK Sian L. Math anxiety: Who has it, why it develops, and how to guard against it. Trends in cognitive sciences, 16 (8): 404-406, julho de 2012. doi: 10.1016 / j.tics.2012.06.008.

MOURA-SILVA, Marcos Guilherme; BENTO-TORRES, João; GONÇALVES, Tadeu Oliver. Manifestações subjacentes da ansiedade matemática no sistema nervoso autônomo: uma análise da Variabilidade da Frequência Cardíaca, Desempenho Matemático e Função Executiva em Crianças Escolares. Belém; UFPA, 2019. Tese de doutorado, Instituto de Educação Matemática e Científica, Universidade Federal Pará, Belém, 2019.

OBERSTEINER, Andreas; TUMPEK, Christine. Measuring fraction comparison strategies with eye-tracking. ZDM Mathematics Education, 48, 255-266, outubro 2016. doi.org/10.1007/ s11858-015-0742-z

OLIVEIRA, Maria Cláudia Santos Lopes de. Narrativas e desenvolvimento da identidade profissional de professores. Cadernos CEDES, Campinas, 32(88),369-378, Dezembro. 2012.

OWENS, Kay. The impact of a teacher education culture-based project on identity as a mathematically thinking teacher. Asia-Pacific Journal of Teacher Education, 42(2), 186-207, abril 2014. Doi: 10.1080 / 1359866X.2014.892568 
ROTZER S; KUCIAN K; MARTIN E; VON Aster M; KLAVER P; LOENNEKER T. Optimized voxel-based morphometry in children with developmental dyscalculia. Neuroimage, 39(1), 417422, janeiro de 2008 doi:10.1016/j.neuroimage.2007.08.045.

RYKHLEVSKAIA, Elena; UDDIN, Lucina Q; KONDOS, Leeza; MENON, Vinod. Neuroanatomical correlates of developmental dyscalculia: combined evidence from morphometry and tractography. Front Hum Neurosci.; 3:51. Novembro 2009. doi:10.3389/neuro.09.051.2009.

SFARD, Anna; PRUSAK, Anna. Telling Identities: In: Search of an Analytic Tool for Investigating Learning as a Culturally Shaped Activity. Educational Researcher, 34(4),14-22, Maio 2005. doi: 10.3102/0013189X034004014

STAVY, R., GOEL, V., CRITCHLEY, H., DOLAN, R. Intuitive interference in quantitative reasoning. Brain Research, 1073-1074, 383-388, fevereiro, 2006. doi: 10.1016 / j.brainres.2005.12.011. .

SUPEKAR, Kaustubh; SWIGART, Anna G..; TENISON, Caitlin; JOLLES, Dietsje D.; ROSENBERG-LEE, Miriam; FUCHS, Lynn; MENON, Vinod. Neural predictors of individual differences in response to math tutoring in primary-grade school children. Proc Natl Acad Sci U S A. 110(20):8230-8235, maio 2013. doi:10.1073/pnas.1222154110

VAN DER MEULEN, A., KRABBENDAM, L., de RUYTER, D. Educational neuroscience: its position, aims and expectations. British Journal of Educational Studies, 63 (2), 229-243, 2015. doi:10.1080/00071005.2015.1036836

WATSON, Cate. Narratives of practice and the construction of identity in teaching. Teachers and Teaching. 12 (5): 509-526, Outubro, 2006. DOI: 10.1080 / 13540600600832213

WENGER, E. Communities of practice: learning, meaning, and identity. Cambridge: Cambridge University Press, 1998.

TAN, Yuen Sze Michelle; AMIEL, Joshua Johnstone. Teachers learning to apply neuroscience to classroom instruction: case of professional development in British Columbia, Professional Development in Education, novembro, 2019. doi: 10.1080/19415257.2019.1689522

ZAMARIAN, L., ISCHEBECK, A.; DELAZER, M. Neuroscience of learning arithmetic-Evidence from brain imaging studies. Neuroscience \& Biobehavioral Reviews, 33(6), 909-925, junho, 2009. doi: https://doi.org/10.1016/j.neubiorev.2009.03.005 